\title{
71. Alteration of Troponin Subunits in Progressive Muscular Dystrophy (DMP). II
}

\section{Mechanism of the Alteration of Troponin Subunits in DMP}

\author{
By Hideo Sugita and Yasuo ToYokura \\ Department of Neurology, Institute of Brain Research, Faculty of Medicine, \\ University of Tokyo, Tokyo, Japan
}

(Comm. by Osamu Hayaishi, M. J. A., May 12, 1976)

It was demonstrated in the previous paper ${ }^{11}$ that the muscles of the Duchenne type of DMP showed the decrease in the TN-I and TN-C components with relative preservation of TN-T even in the relatively early stage of the disease.

The abnormal pattern of troponin subunits was not observed in either neurogenic muscular atrophies or myopathies other than muscular dystrophy so far determined.

The present study was undertaken to investigate the mechanism of the alteration of troponin subunits to shed some lights on the mechanism of the degradation of the structural proteins in DMP.

Materials and methods. Glycerinated muscle fibers of monkey were homogenized with phosphate buffered saline ( $\mathrm{pH}$ 7.0) by Waring Blendor and centrifuged. The residue was suspended in the same solution and centrifuged to remove the soluble proteins. This procedure was repeated once more. The resulting residue was suspended in phosphate buffered saline solution and digested by the addition of 3 or $5 \mu \mathrm{g}$ of trypsin per $\mathrm{mg}$ of protein for 3 or 5 minutes in the water bath at $25^{\circ} \mathrm{C}$ and the reaction was stopped by the addition of trypsin inhibitor, twice the amount of trypsin, and the residue was collected by centrifugation. The native tropomyosin was extracted from this residue by the method as described previously.

$\mathrm{Ca}^{2+}$-activated sarcoplasmic factor (CASF) was extracted from the rabbit skeletal muscle by the method described by Busch et al. in 1972. ${ }^{2}$ The homogenized glycerinated monkey muscle fibers were divided into 5 parts and to each sample was added an amount of CASF one fifth that of myofibrils. Then the Ca ion concentration of each sample was adjusted to less than $10^{-3} \mathrm{M}$ respectively and incubated for 20 hours in the water bath at $25^{\circ} \mathrm{C}$ and the precipitate was collected by centrifugation. The native tropomyosin was extracted from these precipitates by the method as described previously. Each component of troponin was identified by SDS gel electrophoresis. 

$3 \mu \mathrm{gg} / \mathrm{mgr}$
$5 \mathrm{\mu g} / \mathrm{ml}$

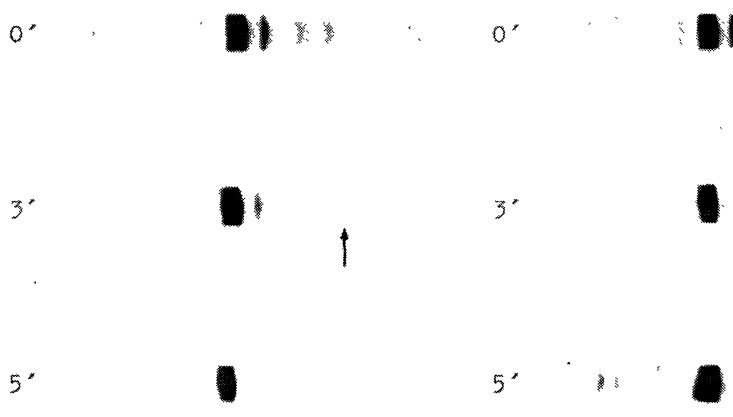

Fig. 1. SDS gel electrophoretic pattern of glycerinated monkey muscle pretreated with 3 or $5 \mu \mathrm{g} / \mathrm{mg}$ of trypsin for 3 or 5 minutes. The arrow indicates the band corresponding to the digested troponin.

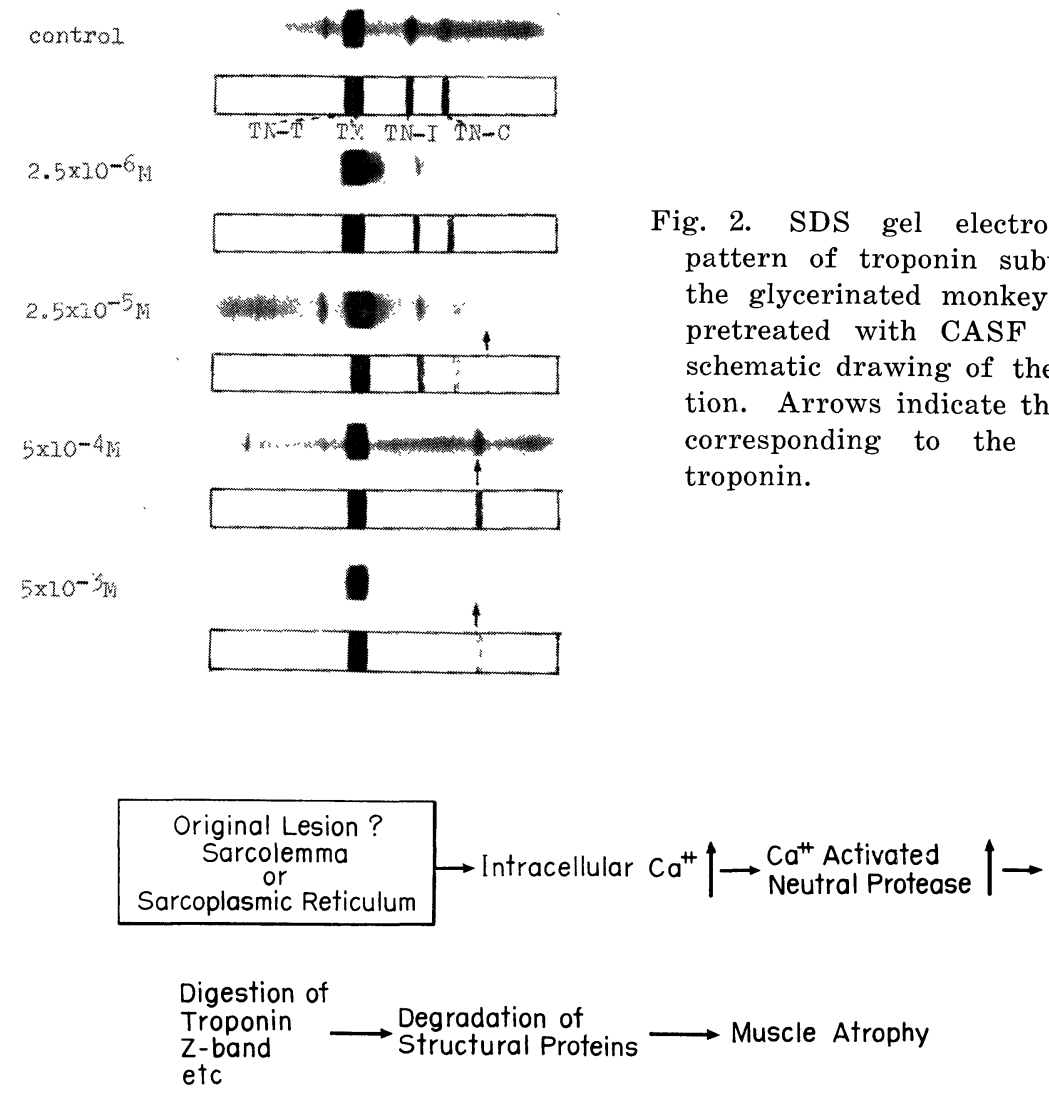

Fig. 3. Schematic illustration of the hypothesis for the degradation process of structural proteins in Duchenne DMP. 
Results. Pattern of the troponin subunits of the glycerinated monkey muscle fibers pretreated with trypsin. Fig. 1 shows the gel electrophoretic pattern of troponin extracted from the muscle fibers pretreated with trypsin in the water bath of $25^{\circ} \mathrm{C}$.

By the treatment of $3 \mu \mathrm{g} / \mathrm{mg}$ of trypsin for 3 minutes the bands of TN-I and TN-C, especially the former, were decreased in their amounts and a band of digested troponin emerged. When treated for 5 minutes, the bands of TN-I and TN-C almost disappeared but TN-T was well preserved. When $5 \mu \mathrm{g} / \mathrm{mg}$ of trypsin was applied for 3 minutes, the bands of both TN-I and TN-C were no longer observed, but a considerable amount of TN-T was still recognized.

Pattern of the troponin subunits of the glycerinated monkey muscle fibers pretreated with CASF. Fig. 2 shows the gel electrophoretic pattern of troponin pretreated with CASF in the presence of various concentrations of $\mathrm{Ca}$ ion. In the presence of $10^{-6} \mathrm{M}$ or less of $\mathrm{Ca}$ ion, the amounts of TN-I and TN-C were normally preserved, but in the presence of $2.5 \times 10^{-5} \mathrm{M} \mathrm{Ca}$ ion, the amounts of TN-I and $\mathrm{TN}-\mathrm{C}$ were decreased with relative preservation of $\mathrm{TN}-\mathrm{T}$, and the band corresponding to the digested troponin began to appear. In the presence of more than $5 \times 10^{-4} \mathrm{M} \mathrm{Ca}$ ion, the bands of TN-I and TN-C were no longer demonstrated and only the band of digested troponin was observed on the gels. However, throughout the whole experiments, the TN-T was relatively well preserved.

Discussions and conclusion. The electrophoretic pattern of the components of troponin extracted from trypsin-digested monkey muscle fibers showed a pattern similar to that observed in Duchenne dystrophic muscle. ${ }^{1)}$ This result strongly suggests that some kind of proteolytic enzyme might play a role in a disappearance of TN-I and TN-C from the dystrophic muscle myofibrils.

According to Ebashi and Nonomura, ${ }^{3)}$ among three components of troponin, TN-T and TN-I are both sensitive to the digestion by trypsin, but TN-C is relatively resistant.

Disappearance of TN-I is well explained by its sensitivity to proteolytic digestion. TN-C has affinities both for TN-I and TN-T in in vitro system,4) but the present results may be interpreted that TN-C is mainly bound to TN-I in situ and removed from myofibrils concomitantly with the digestion of TN-I. The fact that TN-T is well preserved is rather unexpected from its high sensitivity to proteolytic digestion. However, this discrepancy might be explained by the strong affinity of TN-T for tropomyosin in vivo, the susceptible part of TN-T to trypsin being protected by its binding to tropomyosin.

Iodice et al. reported ${ }^{5)}$ that the degradation of the structural proteins was caused by the acidic proteases, i.e., cathepsin and other 
so called lysosomal enzymes which were shown to have a high activity in the affected muscle of dystrophic chicken. But according to the recent observation of Pearson and $\mathrm{Kar}^{6}$ ) in human dystrophic muscles, the cathepsin activity was almost equal to that in neurogenic atrophy and not so markedly increased as reported in dystrophic chicken. So, it is unlikely that the cathepsin played an important role for the degradation of troponin in human muscular dystrophy.

It was demonstrated in the present investigation that the Caactivated sarcoplasmic factor or neutral protease, reported by Busch et al. which is identical with kinase activating factor (KAF) first reported by Meyer et al.7) and Huston and Krebs, ${ }^{8)}$ was also shown to exert essentially the same degrading effect on troponin of monkey glycerinated muscle in the presence of more than $2.5 \times 10^{-5} \mathrm{M} \mathrm{Ca}$ ion as observed in dystrophic muscle.

Therefore, if the sarcolemma and related membraneous system including the sarcoplasmic reticulum is the original site of the lesion in DMP, the increase in the influx of extracellular $\mathrm{Ca}$ ion across the sarcolemma and the release of accumulated $\mathrm{Ca}$ ion from the sarcoplasmic reticulum may increase the intracellular $\mathrm{Ca}$ ion concentration and consequently activate the Ca-activated neutral protease to initiate the degradation of troponin and other structural proteins as illustrated in Fig. 3.

Acknowledgement. We wish to express our cordial thanks to Prof. S. Ebashi for his suggestions and advice on this work. We are also indebted to Miss T. Hirai for her technical assistance. This work was supported in part by a grant from the Ministry of Health and Welfare, Japan, No. 216.

\section{References}

1) Sugita, H., and Toyokura, Y.: Proc. Japan Acad., 52, 256 (1976).

2) Busch, W. A., Stromer, M. H., Goll, D. E., and Suzuki, A.: J. Cell Biol., 52, 367 (1972).

3) Ebashi, S., and Nonomura, T.: The Structure and Function of Muscle. New York and London, Academic Press, 3, 386 (1973).

4) Ebashi, S., Ohtsuki, I., and Mihashi, K.: Cold Spring Harbor Symposium on Quantitative Biology, Vol. 37. The mechanism of muscle contraction. Cold Spring Harbor Laboratory, 215 (1973).

5) Iodice, A. A., Perker, S., and Weinstock, I. M.: Muscle Diseases. Amsterdam, Excerpta Medica Foundation, 313 (1970).

6) Pearson, C. M., and Kar, N. C.: Clinical Studies in Myology. Amsterdam, Excerpta Medica Foundation, 87 (1973).

7) Meyer, W. L., Fischer, E. H., and Krebs, E. G.: Biochemistry, 3, 1033 (1964).

8) Huston, R. B., and Krebs, E. G.: Biochemistry, 7, 2116 (1968). 